[Running title] Outdoor mass cultivation of the oleaginous diatom

\title{
Seasonal Variation of Biomass and Oil Production of the Oleaginous Diatom Fistulifera sp. in Outdoor Vertical Bubble Column and Raceway-type Bioreactors
}

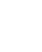

\author{
[Rumning title] Outdoor mass cultivation of the oleaginous diatom
}

7

Reiko Sato ${ }^{1}$, Yoshiaki Maeda ${ }^{1}$, Tomoko Yoshino ${ }^{1}$ Tsuyoshi Tanaka $^{1,2}$ and Mitsufumi

(1)

$$
\text { Matsumoto }^{2,3 *}
$$

${ }^{1}$ Department of Biotechnology, Faculty of Engineering, Tokyo University of Agriculture \& Technology, 2-24-16, Naka-cho, Koganei, Tokyo, 184-8588, Japan ${ }^{2}$ JST, CREST, Sanbancho 5, Chiyoda-ku, Tokyo 102-0075, Japan

${ }^{3}$ Biotechnology Laboratory, Electric Power Development Co., Ltd, 1, Yanagisaki -machi, Wakamatsu-ku, Kitakyusyu, 808-0111, Japan

7

8

19

\section{*Corresponding author:}

Mitsufumi Matsumoto

E-mail: mitsufumi_matsumoto@jpower.co.jp

Tel: +81-93-741-0942

Fax: +81-93-741-0959 


\section{$1 \quad$ Key words}

2 Marine diatom; Fistulifera sp. strain JPCC DA0580; Biodiesel production; Outdoor mass

3 cultivation; Repeated batch culture; Energy conversion efficiency

4

5 
1 Abstract

2 To evaluate the feasibility of industrial biodiesel production, outdoor mass cultivation of the

3 marine oleaginous diatom, Fistulifera sp. strain JPCC DA0580, was conducted in bench-scale

4 photobioreactors ( 200 litters, raceway- and column-types) and seasonal variation of biomass

5 and oil content were monitored. Through three seasons (from spring to autumn), the

6 microalgae showed steady growth and oil accumulation in both reactors in spite of fluctuating

7 temperature and solar irradiation. When comparing the both reactors, the column-type

8 bioreactor was better with regard to energy conversion efficiency compared to the

9 raceway-type bioreactor. The areal oil productivity of $3.23 \mathrm{~g} / \mathrm{m}^{2} /$ day is comparable or even

higher level as compared with the one from other oleaginous microalgae prepared in outdoor

11 mass cultivation. Furthermore, repeated batch culture experiments resulted in success at least

125 cycles. Through the experimental period, little bacterial contamination was observed while

13 protozoal contamination was a fatal issue. The microalgal cell was robust enough to be

14 handled by an automated pump system in inoculation and harvesting processes, and cell

15 adhesion to the bioreactor wall was not observed. These beneficial features could realize ease

16 of oil production and system maintenance. These findings ensure promising innovation by

17 means of outdoor mass cultivation with this strain toward biodiesel production. 


\section{Introduction}

Sustainable energy production without massive release of $\mathrm{CO}_{2}$ is one of the critical issues to be addressed in $21^{\text {st }}$ century due to approaching exhaustion of fossil fuels and their negative impacts on climate. Bioenergy production (e.g., bioethanol, biobuthanol and biodiesel) by using photosynthetic systems of higher plants (e.g., corn, sugar cane, oil palm, and Jatropha) have been recognized as a promising solution to address this issue. However, current bioenergy production from plants is often in competition with food production and requires huge areas of cultivated land due to their low biomass productivity of traditional annual crops, thus discovery of alternative energy producers have been desired. Microalgal biodiesel production is an emerging technology in such pursuits because of their great features (e.g., global carbon dioxide fixation, no competition with food, and much higher biomass yield than higher plants (1)). Most recent studies have revealed that candidate species for biofuel production had diversity and could be found in several microalgal divisions including Chlorophyta , Heterokontophyta (including Bacillariophyceae (so-called diatom) and Eustigmatophyceae), Haptophyta, Rhodophyta and Dinoflagellata (2-5).

We recently discovered an outstanding lipid-producer, Fistulifera sp. strain JPCC DA0580 from our marine microalgal culture collection (6). Beneficial features of this diatom for biodiesel production are high neutral lipid content (40-60\%, w/w (6)) as well as high growth rate (among a range of oleaginous microalgae, some species including Fistulifera sp. strain JPCC DA0580 reach plateau in growth only within 1 week, while others need 2 weeks or more(6-10)); it follows that this strain shows as high yield of oil production as other known oleaginous species, such as Nannochloropsis sp. or Chlorella sp. (2, 7, 11, 12). Fatty acid composition in triacylglycerol from this strain is also suitable for fuel usage because it mainly produces fatty acid methyl esters with low unsaturation degree, such as methyl palmitate (C16:0) and methyl palmitoleate (C16:1), which can generate high quality biodiesel (13). 
1 Recently whole genome analysis of this strain has been launched (14). Based on this genetic

2 information, the transformation technique for this strain has been established (15). These

3 basic studies could allow us to understand the biological mechanisms of high triacylglycerol

4 accumulation in this strain, thereby contributing to improving biodiesel production.

However, thus far, the experiments demonstrating the advantages of this strain for mass

production of biodiesel have been carried out in laboratory-scale ( $\sim 1$ litter). To fully evaluate the potential of Fistulifera sp. as a biodiesel producer compared to other species, and to accommodate huge energy demand in the future, laboratory-scale experiments are not enough and mass cultivation study must take place. Indoor mass cultivation study for this strain has been completed and provided useful information for optimization of culture conditions (16). The next challenge should be an outdoor mass cultivation which could truly make the industrial biodiesel production reality. Outdoor mass cultivation is not a simple expansion of laboratory-scale experiments, and has several difficulties (e.g., risk of bacterial/protozoal contamination, seasonal fluctuation of temperature and sunlight, tight adhesion of algal cell to bioreactor wall and limited usage of gene modified microalgae). To our best knowledge, only a few species has been under the successful investigation in outdoor mass cultivation including; Chlorella, with biodiesel production rates of $0.7 \mathrm{~g} \mathrm{~L}^{-1}$ day ${ }^{-1}$ in $8,000 \mathrm{~L}$ stirred tank bioreactor under heterotrophic condition (17); Nannochloropsis, with biomass productivity of $0.15 \mathrm{~g} \mathrm{~L}^{-1}$ day $^{-1}$ in 20-60 L outdoor flat-panel bioreactor (18); Tetraselmis, with oil productivity of $0.05 \mathrm{~g} \mathrm{~L}^{-1}$ day $^{-1}$ in $16 \mathrm{~L}$ outdoor Green Wall panels (19).

In this study, cultivation of Fistulifera sp. with 200-liter bench-scale bioreactor was carried out to clarify the unique features of this strain under outdoor mass cultivation. Two distinct types of open bioreactors were used to determine better design for high oil content. The contamination issue was also examined by using a non-sterile medium. These results provided new insights in mass cultivation of Fistulifera sp. toward efficient biodiesel production. 
Materials and Methods

3

\section{Photobioreactor design}

Two distinct types of photobioreactors, a raceway-type and a vertical column-type, were used (Fig. 1). Both reactors are made of acrylic substrates. The raceway-type bioreactor has a racetrack with a width of $22.5 \mathrm{~cm}$, and equips a waterwheel $(30 \mathrm{rpm})$ for circulation of medium and four triangular objects at the bottom of the track for efficient agitation. The vertical column-type bioreactor has a tubular structure with a height of $2 \mathrm{~m}$ and a diameter of $40 \mathrm{~cm}$ in which air blows $(6 \mathrm{~L} / \mathrm{min}$ over incubation period) from the bottom of the column was conducted in order for effective agitation with assistance from a stirrer system (160 rpm).

\section{Algal strain and culture conditions}

The marine oleaginous diatom, Fistulifera sp. strain JPCC DA0580 (formerly Navicula sp. strain JPCC DA0580 (6)), was obtained from J-Power Culture Collection. Seed culturing was done in sterile f/2 medium (20) (a common medium for diatoms, 75-mg $\mathrm{NaNO}_{3}, 6-\mathrm{mg}$ $\mathrm{Na}_{2} \mathrm{HPO}_{4} \cdot 2 \mathrm{H}_{2} \mathrm{O}, \quad 0.5-\mu \mathrm{g}$ vitamin $\mathrm{B}_{12}, \quad 0.5-\mu \mathrm{g}$ biotin, $100-\mu \mathrm{g}$ thiamine $\mathrm{HCl}, 10-\mathrm{mg}$ $\mathrm{Na}_{2} \mathrm{SiO}_{3} \cdot 9 \mathrm{H}_{2} \mathrm{O}$, 4.4-mg $\mathrm{Na}_{2}$-EDTA, 3.16-mg $\mathrm{FeCl}_{3} \cdot 6 \mathrm{H}_{2} \mathrm{O}, 12-\mu \mathrm{g} \mathrm{CoSO}_{4} \cdot 5 \mathrm{H}_{2} \mathrm{O}, 21-\mu \mathrm{g}$ $\mathrm{ZnSO}_{4} \cdot 7 \mathrm{H}_{2} \mathrm{O}, 0.18-\mathrm{mg} \mathrm{MnCl}_{2} \cdot 4 \mathrm{H}_{2} \mathrm{O}, 70-\mu \mathrm{g} \mathrm{CuSO} \cdot 5 \mathrm{H}_{2} \mathrm{O}$, and $7-\mu \mathrm{g} \mathrm{Na}_{2} \mathrm{MoO}_{4} \cdot 2 \mathrm{H}_{2} \mathrm{O}$ ) that was dissolved in a liter of artificial seawater (Tomita Pharmaceutical Co., Ltd., Tokushima, Japan). Cultures were grown in closed flat-shaped flasks $(500 \mathrm{~mL})$ at $24^{\circ} \mathrm{C}$ under continuous, cool-white fluorescent lights at $100 \mu \mathrm{mol} \cdot \mathrm{m}^{-2} \cdot \mathrm{s}^{-1}$ for $5-7$ days under $1 \% \mathrm{CO}_{2}$ at $25^{\circ} \mathrm{C}$ in a rotary shaker at $90 \mathrm{rpm}$, and then used as a seed culture for mass cultivation experiments.

For outdoor mass cultivation, a serial scale-up strategy was employed where the seed culture (500 mL) was diluted in fresh, non-sterile f/2 medium (40 L) prepared by using tap water, and incubated outdoors until stationary phase, subsequently another dilution was done in the raceway-type or column-type bioreactor including the same medium $(200 \mathrm{~L})$. The bioreactors 
1 were placed at Kitakyusyu (3392'73'N, 130 74 '15'E), Japan over spring of 2011 to autumn

2 of 2012 with a break period in winter. During the summer and autumn months

3 (August-November) of 2011, repeated batch culture was carried out as below. The microalgae

4 were incubated for 7-10 days. A part of resulting culture was harvested. Then $50 \mathrm{~L}$ of

5 remaining culture was used as the seed culture for next batches. During the experimental

6 period, solar irradiation and ambient temperature were monitored using a photovoltaic power

7 generation system. The culture medium used in mass cultivation was handled by using an

8 automated pump system.

9 Quantification of cellular oil content

Algal oil, mainly neutral lipids, was extracted by disrupting the lyophilized cells ( $c a .100$ $\mathrm{mg})$ in $2 \mathrm{ml} n$-hexane with zirconia beads $(0.7 \mathrm{~mm}$ diameter; BioSpec Products, Oklahoma, USA) using a beads cell disrupter (Micro Smash MS-100R, TOMY, Tokyo, Japan) at 5,000 $\mathrm{rpm}$ for $5 \mathrm{~min}$ at $4^{\circ} \mathrm{C}$. The resultant homogenate was then centrifuged at $20,000 \times \mathrm{g}$ for $5 \mathrm{~min}$ at $4{ }^{\circ} \mathrm{C}$ and the supernatant obtained was transferred to a glass vial as an algae oil fraction. This extraction/fractionation was repeated four times. The microalgal oil fraction pooled was then concentrated to dryness and weighed. The cellular oil content was expressed as percentage of dry algal mass (w/w).

\section{Energy conversion efficiency}

In order to evaluate how efficiently the solar energy was converted into chemical energy in production of oleaginous biomass, energy conversion efficiency (ECE, $\eta$ ) was calculated by using the following equation.

$$
\eta=E_{\text {out }} / \mathrm{E}_{\text {in }}=\mathrm{cW} / \mathrm{IAt}
$$

where $\mathrm{E}_{\text {out }}$ is energy produced from microalgal biomass, $\mathrm{E}_{\text {in }}$ is solar energy absorbed in each photobioreactor, c is energy content of Fistulifera sp. (26.9 MJ/kg) (6), W is final value of biomass produced in photobioreactors $(\mathrm{kg})$, I is average solar irradiation during each 
1 incubation period, $\mathrm{A}$ is irradiation area for each bioreactor. Irradiation area of the

2 raceway-type bioreactor is $1 \mathrm{~m}^{2}$ (total area of the racetrack) and the one of the column-type

3 bioreactor is $0.8 \mathrm{~m}^{2}$ (cross-section area of the column). $\mathrm{t}$ is incubation time (h).

4

5

6

7

8

\section{Results and discussion}

\section{Mass cultivation and repeated batch cultures in the bioreactors}

Toward mass production of biodiesel, stable outdoor cultivation is the critical criteria to be fulfilled. For investigation of this issue, bench-scale cultivation ( 200 L) of Fistulifera sp. strain JPCC DA0580 in the raceway-type and the column-type bioreactors was carried out in which the growth medium was not sterilized and temperature was uncontrolled.

Seasonal biomass concentration and oil content throughout the whole experimental period (April 2011-November 2012) were summarized in Table 1. Through three seasons (spring-autumn), mass cultivation of this strain was successfully achieved with high level of oil accumulation in both reactors. Even in summer, steady growth of the microalgae was confirmed despite there were several days when average daily ambient temperature exceeded $30^{\circ} \mathrm{C}$ (Fig. S1) and it temporarily reached $42^{\circ} \mathrm{C}$, thus demonstrating the thermostable feature of this strain. In contrast, winter season (from November to March) is not suitable because significant decrease in growth rate of Fistulifera sp. was observed when ambient temperature was as low as $15^{\circ} \mathrm{C}$. Therefore heat supply and/or usage of alternative species that is tolerant of low temperature should be considered to realize year-round production. The cultivation systems employed in this study are further scalable by expanding the reactor size (especially for the raceway-type reactor)(21) or by placing many reactors in the optimized arrangement (22).

When comparing the raceway-type and the column-type bioreactors, there is no significant difference with regard to biomass concentration (Fig. 2A). In contrast, large difference exists 
1 in oil content; the algal oil content in the column reactor was higher than the one from the

2 raceway bioreactor (Fig. 2B), and maintained as high level as laboratory-scale experiment

$3(\sim 40 \%)$ (6). This could be due to the air supply performed in the column reactor. The air

4 bubbling $(6 \mathrm{~L} / \mathrm{min})$ contributes to the better agitation effect, by which each microalgal cell

could be exposed to stronger light supply. Strong light stress is one of the typical switches

which can induce the intensive increase of the oil productivity in microalgae (23).

Areal oil productivity was calculated (Table S1) based on the dimension of bioreactors (Fig.

1), biomass concentration and oil content (Table 1). The highest areal oil productivity was recorded as $3.23 \mathrm{~g} / \mathrm{m}^{2} /$ day in the spring and summer of 2012 using the vertical column-type bioreactor at Kitakyusyu. This value is comparable or even higher oil productivity compared with other promising oil producer (outdoor mass cultivation of Tetraselmis suecica F\&M-M33 in Italy by using Green Wall Panel reactor (2) including filtered medium; temperature was controlled by water-cooling system not to exceed $25^{\circ} \mathrm{C}$; $\mathrm{pH}$ was also maintained by $\mathrm{CO}_{2}$ blows.) (19). This oil production yield from Fistulifera sp. would further be improved by optimization of culturing media along with indoor mass cultivation, so that this strain can provide more promising feasibility for mass production of biodiesel (16). Energy conversion efficiencies $(\eta)$ in the raceway-type and column-type bioreactors throughout the experimental period were revealed to be stable with the average values of $1.9 \pm 0.7 \%$ and $3.1 \pm 0.7 \%$ respectively. Difference of these two values corresponds to higher oil content in the column bioreactor (Table 1). It should be noted that when the energy conversion efficiencies were calculated in this study, the energy content of this microalgal strain was approximated to be $26.9 \mathrm{MJ} / \mathrm{kg}$ according to our previous work in which the oil content was $\sim 40 \%$ of dry cell weight(6). However, the energy content can vary depending on the oil content. In this study, the oil content of microalgal biomass in the column-type reactor was as high as the previous work, while that in the raceway was lower; it follows that the 
actual energy conversion efficiency in the raceway reactor may be even lower than our calculation. Altogether, we could conclude that the vertical column-type bioreactor demonstrated higher performance than the raceway reactor for outdoor cultivation of Fistulifera sp. in the analyzed conditions. This would not be surprising because the column-type reactor equips the air supply system, which contributes to better agitation effect and $\mathrm{pH}$ stability. It is predictable that the raceway bioreactor can also be improved by using same systems(24), while energy consumption by the air supply system would decline the whole energy production efficiency. We will further investigate its impact in the future projects.

Repeated batch cultures were also attempted (August -November 2012) using this strain. Repeated batch culture is a mass culturing technique where batch culture is repeated by using a part of the former batch as a seed of the next batch, and this can shorten whole incubation time due to high biomass concentration at the initial culturing stage. In this study, approximately $200 \mathrm{~L}$ of medium was incubated in the photobioreactors, a part of resulting culture was harvested after 7-10 days incubation, leftover was then used for the next batch process. In this bench-scale test with repeated batch culturing (two sets of 5 cycles in the raceway-type bioreactor and 7 cycles in the column-type bioreactor), the microalgae showed steady growth (Fig. 3). The final biomass concentration varied ranging from 0.2 to $0.4 \mathrm{~g}$ dry weight/L depending on the temperature variation $\left(12.6-31.8^{\circ} \mathrm{C}\right)$. For instance, low biomass concentration was observed in August and early September. The average temperature in this period was $28.8^{\circ} \mathrm{C}$ which exceeded the optimal temperature $\left(25^{\circ} \mathrm{C}\right)$ for the growth of this strain according to the indoor cultivation experiments (16). Low biomass concentration was also observed in November (average temperature, $13.8^{\circ} \mathrm{C}$ ), which is caused by too low temperature. Although these biomass variation depending on the temperature fluctuation was confirmed as is observed in the indoor cultivation study, the successful cycling of the repeated 
batch cultures was demonstrated by using this strain.

\section{Contamination, adhesion observation and cell robustness}

Not only growth rates and oil content but some other factors could greatly affect mass cultivation efficiency. Here we discuss contamination, cell adhesion and cell robustness features of Fistulifera sp. that might inhibitory influence to mass cultivation.

In this study, little bacterial contamination was observed in the culture and microalgal biomass increased steadily despite the usage of unsterilized media and open outdoor photobioractors. This result suggests that Fistulifera sp. could take advantage of high growth rate to suppress bacterial overgrowth, and this feature is greatly beneficial for outdoor mass cultivation. Seawater-based $\mathrm{f} / 2$ medium, in which marine microalgae could grow but non-halophilic microorganisms would be inactivated, could also contribute to inhibit bacterial contamination. In contrast, protozoal contamination was observed in the raceway type reactor once in summer of 2011. Therefore, somehow the protozoal contamination should be eliminated by further studies.

Tight adhesion of algal cells onto the surface of photobioreactors is a well-known problem in mass cultivation. Several microalgae tend to secrete sticky extracellular matrix made from carbohydrates in order to form biofilm and it causes the tight adhesion of microalgal cells to wide range of materials $(25,26)$. Accumulation of microalgal fouling on the bioreactor wall blocks sunlight and decrease photosynthesis efficiency; it directly affects biomass yield and production cost. In this study, no cell adhesion appeared on the surface of the photobioreactors made of acrylic substrates throughout the experiment period (Fig. S2). This result indicates that Fistulifera sp. showed low adhesion character, thus light permeability through the wall of the bioreactor would be guaranteed over cultivation.

When mass cultivation is conducted, it is necessary to handle huge volume of culture media by using an automated pump system for rapid operation. In this process, microalgal cells 
undergo a substantial level of shear stress. This would be harmful to microalgal growth. To test this issue, all culture media including Fistulifera sp. cells were handled using an automated pump system. As a result, no growth inhibition was observed, thus demonstrating that the cell structure of this diatom is robust enough to tolerate the share stress through pump handling. In contrast, excess robustness may prohibit the cell-breaking process, which could result in inefficient oil extraction. To address this concern, the oil productivity in the outdoor bioreactor and laboratory-scale experiments was compared. If the cell robustness in outdoor cultivation significantly increases, extracted oil amount would drastically decrease. However significant decrease in oil productivity was not observed (Table 1, see also our previous work (6)). These results strongly indicate that Fistulifera $\mathrm{sp}$. has appropriate cell robustness which is suitable for oil production and extraction based on outdoor mass cultivation. In conclusion, outdoor mass cultivation of the oleaginous marine diatom, Fistulifera sp. strain JPCC DA0580 was studied by using 200-litter bench-scale bioreactors. As a result, several advantageous features were revealed, such as stable growth through three seasons (spring-autumn) without lacking oil accumulation, fast growth that can maintain low contamination level, low adhesion character and appropriate cell robustness. The growth medium for this strain was able to be prepared from tap water, and sterilization process was not required, thus the system could be readily maintained. These beneficial phenotypes could make this strain a promising candidate for outdoor mass production of biodiesel.

\section{Acknowledgements}

This study was supported by JST, CREST. 
References

3

1. Smith, V. H., Sturm, B. S., Denoyelles, F. J., Billings, S. A.: The ecology of algal biodiesel production, Trends Ecol. Evol., 25, 301-309 (2010).

2. Rodolfi, L., Chini Zittelli, G., Bassi, N., Padovani, G., Biondi, N., Bonini, G., Tredici, M. R.: Microalgae for oil: strain selection, induction of lipid synthesis and outdoor mass cultivation in a low-cost photobioreactor, Biotechnol. Bioeng., 102, 100-112 (2009).

3. Oh, S. H., Han, J. G., Kim, Y., Ha, J. H., Kim, S. S., Jeong, M. H., Jeong, H. S., Kim, N. Y., Cho, J. S., Yoon, W. B., Lee, S. Y., Kang do, H., Lee, H. Y.: Lipid production in Porphyridium cruentum grown under different culture conditions, J. Biosci. Bioeng., 108, 429-434 (2009).

4. Mahapatra, D. M., Chanakya, H., Ramachandra, T.: Euglena sp. as a suitable source of lipids for potential use as biofuel and sustainable wastewater treatment, J. Appl. Phycol., 1-11 (2013).

5. Fuentes-Grunewald, C., Garces, E., Rossi, S., Camp, J.: Use of the dinoflagellate Karlodinium veneficum as a sustainable source of biodiesel production, J. Ind. Microbiol. Biotechnol., 36, 1215-1224 (2009).

6. Matsumoto, M., Sugiyama, H., Maeda, Y., Sato, R., Tanaka, T., Matsunaga, T.: Marine diatom, Navicula sp. strain JPCC DA0580 and marine green alga, Chlorella sp. strain NKG400014 as potential sources for biodiesel production, Appl. Biochem. Biotechnol., 161, 483-490 (2010).

7. Xiong, W., Li, X., Xiang, J., Wu, Q.: High-density fermentation of microalga Chlorella protothecoides in bioreactor for microbio-diesel production, Appl. Microbiol. Biotechnol., 78, 29-36 (2008). 
1 8. Rocha, J. M., Garcia, J. E., Henriques, M. H.: Growth aspects of the marine

2 microalga Nannochloropsis gaditana, Biomol. Eng., 20, 237-242 (2003).

3

4

9. Liang, Y., Sarkany, N., Cui, Y.: Biomass and lipid productivities of Chlorella vulgaris under autotrophic, heterotrophic and mixotrophic growth conditions, Biotechnol. Lett., 31, 1043-1049 (2009).

\section{Casadevall, E., Dif, D., Largeau, C., Gudin, C., Chaumont, D., Desanti, O.:}

Studies on batch and continuous cultures of Botryococcus braunii: hydrocarbon production in relation to physiological state, cell ultrastructure, and phosphate nutrition, Biotechnol. Bioeng., 27, 286-295 (1985).

11. Illman, A. M., Scragg, A. H., Shales, S. W.: Increase in Chlorella strains calorific values when grown in low nitrogen medium, Enzyme. Microb. Technol., 27, 631-635 (2000).

12. Kojima, E., Zhang, K.: Growth and hydrocarbon production of microalga Botryococcus braunii in bubble column photobioreactors, J. Biosci. Bioeng., 87, 811-815 (1999).

13. Knothe, G.: Analyzing biodiesel: standards and other methods, J. Am. Oil Chem. Soc., 83, 823-833 (2006).

14. Tanaka, T., Fukuda, Y., Yoshino, T., Maeda, Y., Muto, M., Matsumoto, M., Mayama, S., Matsunaga, T.: High-throughput pyrosequencing of the chloroplast genome of a highly neutral-lipid-producing marine pennate diatom, Fistulifera sp. strain JPCC DA0580, Photosynth. Res., 109, 223-229 (2011).

15. Muto, M., Fukuda, Y., Nemoto, M., Yoshino, T., Matsunaga, T., Tanaka, T.: Establishment of a genetic transformation system for the marine pennate diatom Fistulifera sp. strain JPCC DA0580--a high triglyceride producer, Mar. Biotechnol., 15, 48-55 (2013). 
1 16. Satoh, A., Ichii, K., Matsumoto, M., Kubota, C., Nemoto, M., Tanaka, M.,

2 Yoshino, T., Matsunaga, T., Tanaka, T.: A process design and productivity

3 evaluation for oil production by indoor mass cultivation of a marine diatom,

$4 \quad$ Fistulifera sp. JPCC DA0580, Bioresour. Technol., 137, 132-138 (2013).

$5 \quad$ 17. Li, X. F., Xu, H., Wu, Q. Y.: Large-scale biodiesel production from microalga

6 Chlorella protothecoides through heterotropic Cultivation in bioreactors,

7 Biotechnol. Bioengineering., 98, 764-771 (2007).

8 18. Quinn, J. C., Yates, T., Douglas, N., Weyer, K., Butler, J., Bradley, T. H.,

9 Lammers, P. J.: Nannochloropsis production metrics in a scalable outdoor photobioreactor for commercial applications, Bioresour. Technol., 117, 164-171 (2012).

19. Bondioli, P., Della Bella, L., Rivolta, G., Chini Zittelli, G., Bassi, N., Rodolfi, L., Casini, D., Prussi, M., Chiaramonti, D., Tredici, M. R.: Oil production by the marine microalgae Nannochloropsis sp. F\&M-M24 and Tetraselmis suecica F\&M-M33, Bioresour. Technol., 114, 567-572 (2012).

20. Guillard, R. R.: Culture of phytoplankton for feeding marine invertebrates, Culture of marine invertebrate animals. Plenum, 29-60 (1975).

21. Spolaore, P., Joannis-Cassan, C., Duran, E., Isambert, A.: Commercial applications of microalgae, J. Biosci. Bioeng., 101, 87-96 (2006).

22. Chinnasamy, S., Bhatnagar, A., Claxton, R., Das, K. C.: Biomass and bioenergy production potential of microalgae consortium in open and closed bioreactors using untreated carpet industry effluent as growth medium, Bioresour. Technol., 101, 6751-6760 (2010).

23. Sharma, K. K., Schuhmann, H., Schenk, P. M.: High lipid induction in microalgae for biodiesel production, Energies, 5, 1532-1553 (2012). 
1 24. Ketheesan, B., Nirmalakhandan, N.: Modeling microalgal growth in an

2 airlift-driven raceway reactor, Bioresour. Technol., 136, 689-696 (2013).

3 25. Irving, T. E., Allen, D. G.: Species and material considerations in the

4 formation and development of microalgal biofilms, Appl. Microbiol. Biotechnol., 92, $5 \quad 283-294(2011)$.

6 26. Rindi, F., Guiry, M. D.: Composition and spatial variability of terrestrial algal 7 assemblages occurring at the bases of urban walls in Europe, Phycologia, 43, $8 \quad 225-235(2004)$.

9

\section{Figure legends}

Fig. 1 The raceway-type (A\&B) and the vertical column-type (C\&D) bioreactors used in this study. The unit indicating size is centimeter.

Fig. 2 Biomass concentration (A) and oil content (B) of Fistulifera sp. strain JPCC DA0580 plotted against solar irradiation. The microalgae were cultured in the raceway-type (closed circle) and vertical column-type (open circle) bioreactors.

Fig. 3 Repeated batch cultures of Fistulifera sp. strain JPCC DA0580 using the raceway (closed circle) and vertical column (open circle) photobioreactors. Ambient temperature (open square) and solar irradiation (closed square) were also monitored. The experimental period was 16 Aug. 2011 to 16 Nov. 2011. 

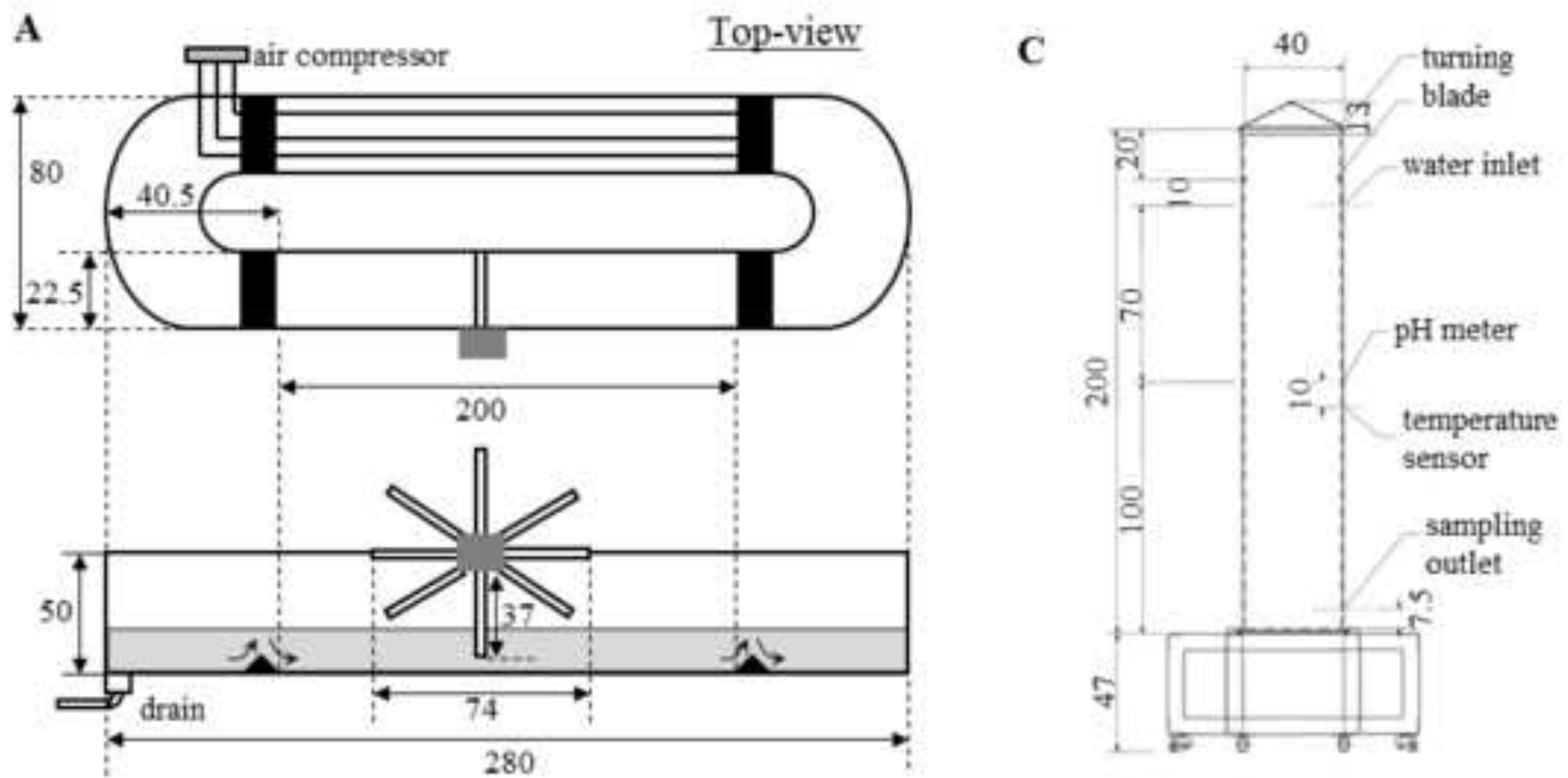

Side-view
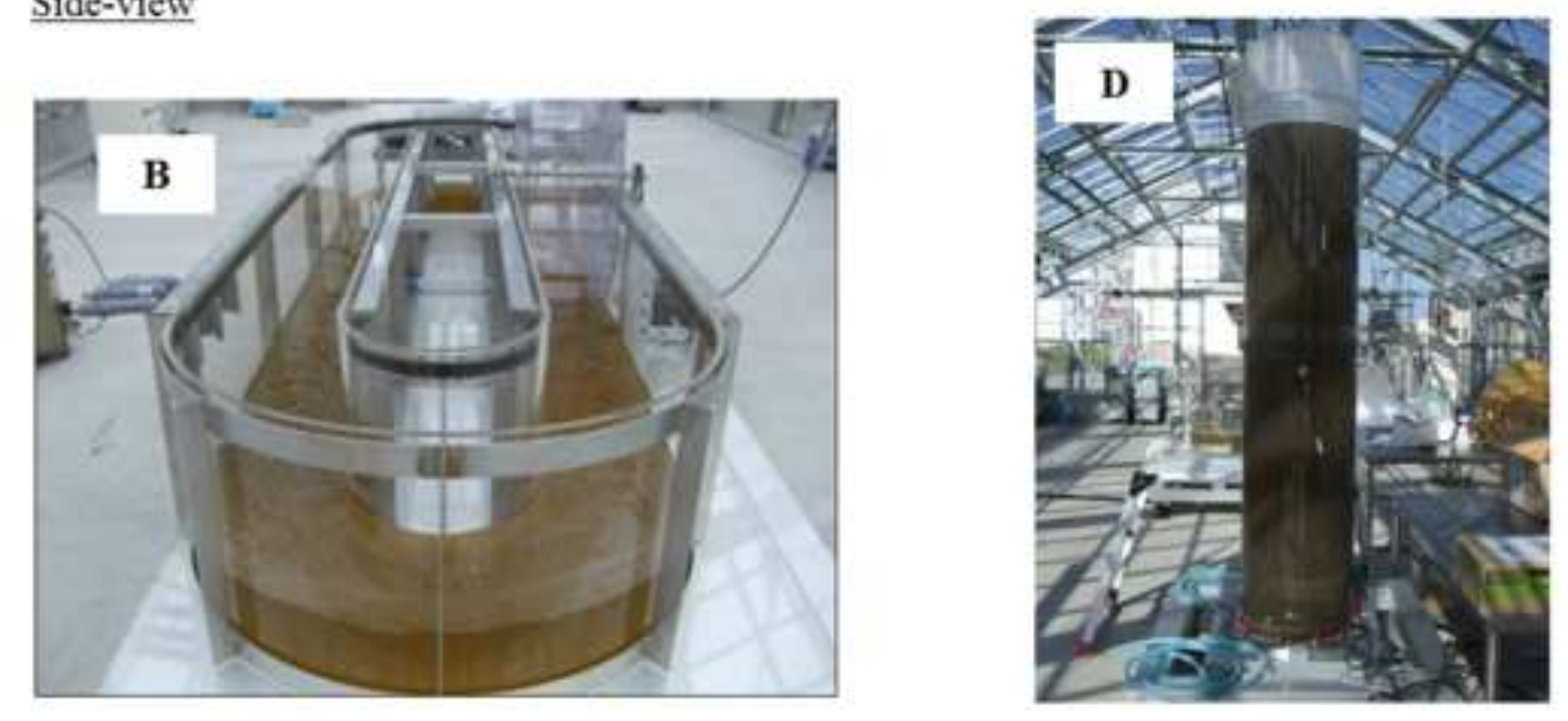
A

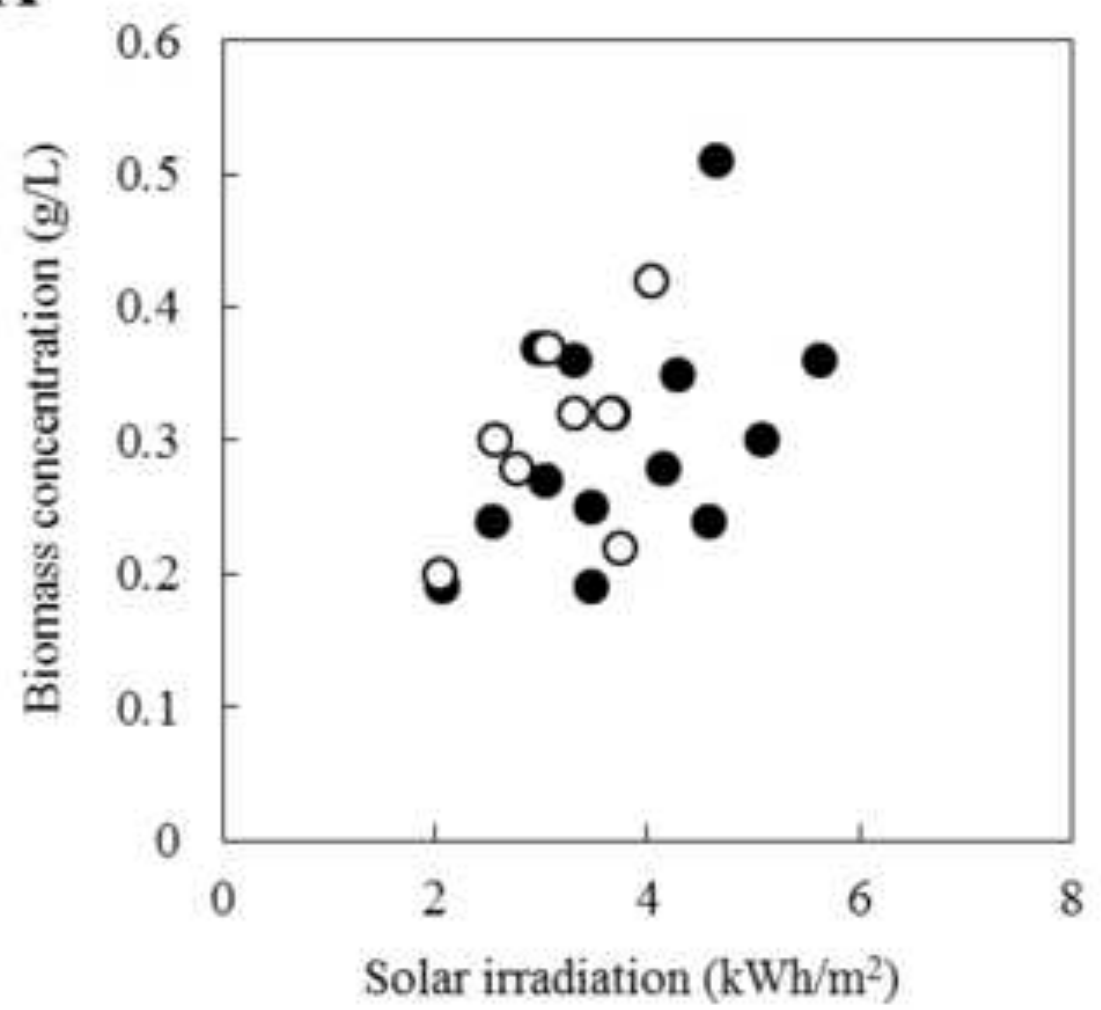

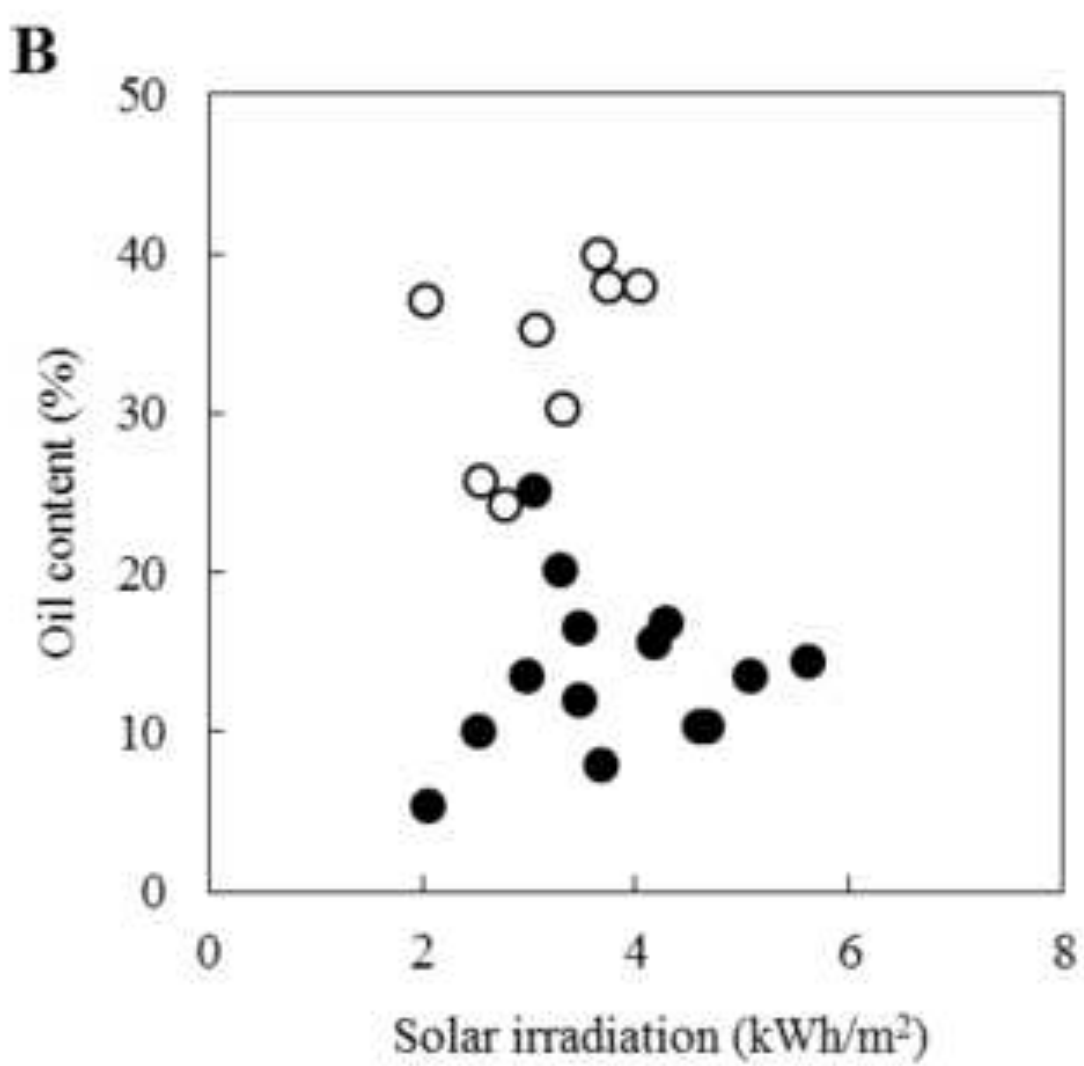



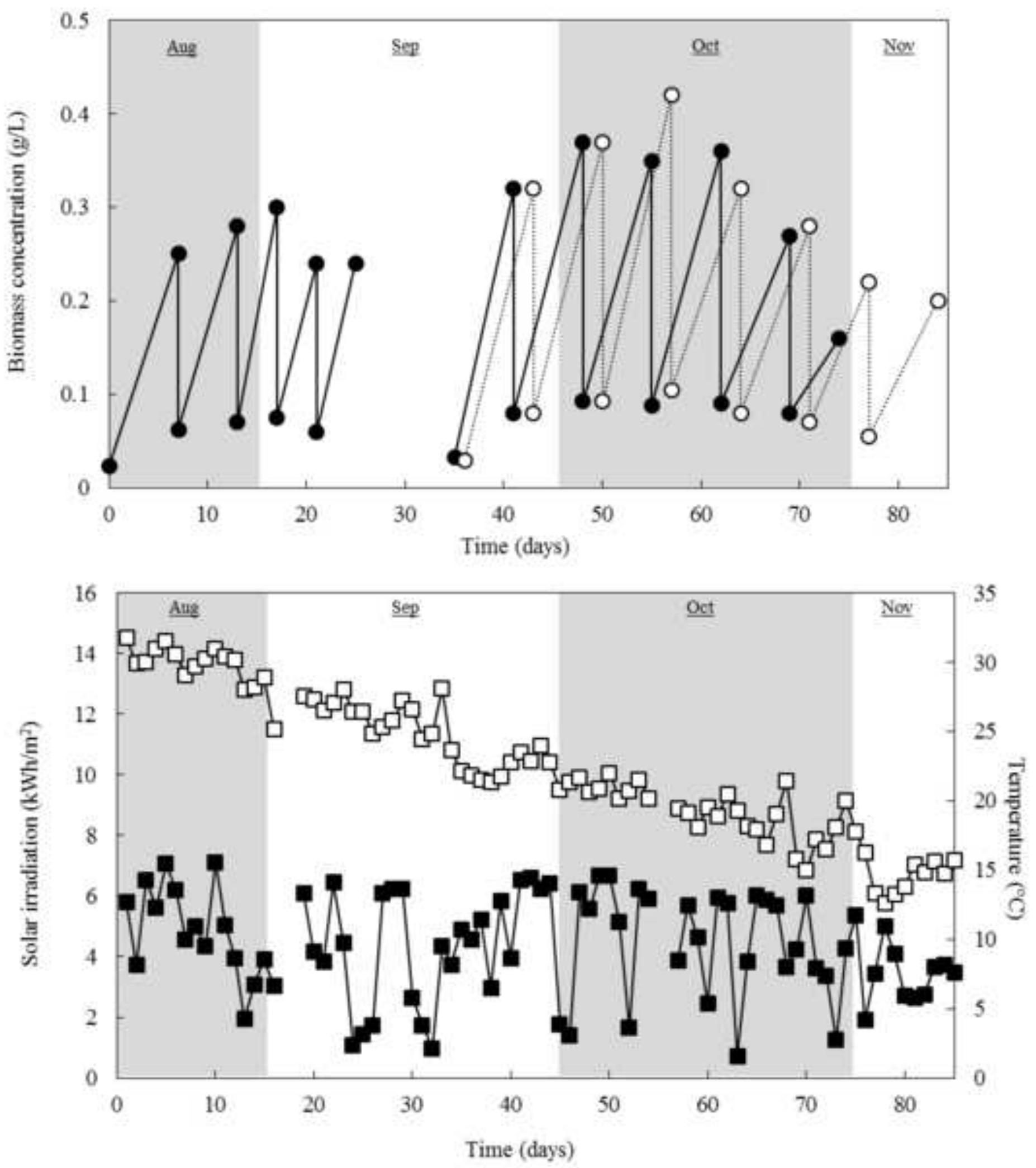
Table 1

Biomass concentration and oil content of Fistulifera sp. strain JPCC DA0580 using the raceway-type and the vertical column-type bioreactors throughout the experimental period

\begin{tabular}{|c|c|c|c|c|}
\hline & \multicolumn{2}{|c|}{ Raceway } & \multicolumn{2}{|c|}{ Vertical column } \\
\hline & Biomass concentartion $(\mathrm{g} / \mathrm{L})$ & Oil content $(\%)$ & Biomass concentartion $(\mathrm{g} / \mathrm{L})$ & Oil content $(\%)$ \\
\hline \multicolumn{5}{|l|}{ Spring } \\
\hline (Apr. - Jun., 2011) & $0.21 \pm 0.02$ & $8.9 \pm 3.9$ & - & - \\
\hline (May. - Jun., 2012) & $0.18 \pm 0.04$ & $17.3 \pm 2.4$ & $0.42 \pm 0.08$ & $33.8 \pm 12.8$ \\
\hline \multicolumn{5}{|l|}{ Summer } \\
\hline (Jul. - Sep., 2011) & $0.32 \pm 0.09$ & $12.5 \pm 2.9$ & $0.35^{\mathrm{a}}$ & $37.7^{\mathrm{a}}$ \\
\hline (Jul. - Sep., 2012) & $0.31 \pm 0.04$ & $14.8 \pm 6.5$ & $0.57 \pm 0.13$ & $17.8 \pm 4.8$ \\
\hline \multicolumn{5}{|l|}{ Autumn } \\
\hline (Oct. - Nov., 2011) & $0.33 \pm 0.05$ & $20.7 \pm 4.1$ & $0.29 \pm 0.09$ & $33.5 \pm 6.1$ \\
\hline (Oct. - Nov., 2012) & - & - & $0.50 \pm 0.19$ & $20.4 \pm 9.1$ \\
\hline
\end{tabular}

a Experiments were repeated twice in this season, thus standard deviation was not calculable. 